\title{
Методы аппроксимации и количественной оценки издержек финансовой неустойчивости
}

\author{
Нефёдов Д.А. ${ }^{14}$
}

В данной статье рассматривается влияние издержек финансовой неустойчивости компании на ее иелевой уровень долга и, как результат, на общую стоимость компании. Также будет проведен обзор существующих работ по выбранной тематике, в которых были сделаны попытки количественной оченки COFD и аппроксимации значения потерь COFD для компаний. Mbl приходим к выводу, что оба типа издержек достигают значительных размеров в финансово несостоятельных фирмах, причем прямые издержки составляют 3-4,5\%, а косвенные - 8-10\%. Значения для прямых COFD практически не колеблются на протяжении времени (начиная с ранних исследований и по сей день), а также по различным отраслям экономики. Напротив, косвенные издержки вызывают больше вопросов, поскольку они трудно наблюдаемы в реальности. Ранее проведеннье эмпирические тесты (в подавляющем больиинстве работ - путем регрессионного анализа), возможно, смогут пролить свет на проблему влияния неявных COFD, поэтому они также включены в наш обзор.

\section{$J E L: G 33$}

Ключевые слова: финансовая неустойчивость, долг, рычаг, банкротство

В стремлении максимизировать общую стоимость компаниям необходимо обращать особое внимание на структуру финансирования своей деятельности. Выбор оптимальной структуры финансирования является основополагающим финансовым решением компании, вне зависимости от приверженности руководства компании к той или иной теории структуры капитала или отсутствия каких-либо предпочтений в принципе. В любом случае, как бы ни оценивалась структура капитала, существующие основные теории (компромиссная, порядковая, сигнальная) лишь расставляют приоритеты одних факторов над другими, но ни в коем случае не пренебрегают последними. В этом смысле понимание и оценка таких явлений компромиссной модели, как, например, издержки финансовой неустойчивости (далее COFD), крайне необходима для всех компаний, использующих заемный капитал, и их обсуждение не должно ограничиваться лишь теорией компромиссного выбора.

Природа COFD и их влияние на решения относительно структуры капитала активно обсуждается в научной среде с 70-х годов, но по-прежнему общий подход к анализу COFD не выработан. В соответствии с традиционным подходом, с определенного момента привлечение долгового финансирования сопровождается неприемлемо высокими издержками потенциального банкротства, и фирмам необходимо либо задуматься о других источниках финансирования, либо умерить свои амбиции роста. Другими словами, эффект давления со стороны издержек финансовой неустойчивости заставляет компании снижать целевой уровень долга. Количественные же оценки COFD основаны в подавляющем большинстве на данных западных компаний, которые уже прошли процедуру реорганизации в законодательном порядке, а значит, эти данные имеют характер ex post. Более того, как мы увидим позже, оценки экспертов значительно колеблются. В то же время менеджерам и инвесторам необходима информация в режиме реального времени; другими словами, нужны достоверные переменные (proxies) для своевременной оценки изменения COFD и их негативного влияния на значение оптимального эффекта рычага. В данной статье мы сначала

\footnotetext{
${ }^{14}$ Студент магистратуры ГУ ВШЭ (группа 61 СУФФ), стажер Научно-учебной лаборатории корпоративных финансов ГУ ВШЭ.
}

Выпуск \#3(7), 2008

(C) Электронный журнал Корпоративные Финансы, 2008 
рассмотрим работы авторов по количественной оценке издержек финансовой неустойчивости, затем проведем обзор существующих исследований в области оценки вероятности банкротства фирмы и ее влияния на стоимость. В итоге мы сделаем качественные выводы по проделанной работе.

Очевидно, что потенциальные издержки банкротства заставляют компании снижать свой целевой уровень долга. Каков масштаб COFD и какова плата за чрезмерное долговое финансирование? Обзор статей на данную тематику, как и ожидалось, указывает на различные результаты. Итак, рассмотрим несколько статей различных авторов, чтобы попытаться составить наиболее полное представление о роли издержек финансовой неустойчивости. Одним из последних исследований по вопросу COFD является работа Бранча [Branch, 2002]. В ней проводится подробный анализ прямых и косвенных издержек банкротства, по компонентам рассматривается их объем как для самой фирмы, так и для лиц, участвующих в распределении собственности (claimsholders). По итогам анализа автор приходит к заключению, что суммарные издержки финансовой несостоятельности для фирмы составляют от 9,45 до $16,35 \%$ от стоимости компании до возникновения финансовой несостоятельности. При этом лица, участвующие в распределении собственности, несут потери (издержки мониторинга и потеря ликвидности активов) в размере $3,25-4,15 \%$ от той же величины. В конечном итоге получается, что суммарные издержки банкротства при различных сценариях могут составлять $12,7-20,5 \%$ от стоимости компании, что весьма значительно. Аналогичные результаты были получены ранее в работе Альтмана [Altman, 1984], где суммарные COFD составили примерно $15 \%$ от стоимости компании до возникновения финансовой несостоятельности для промышленных компаний и 7\% - для сектора розничной торговли. В работе Фрэнкса и Topoca [Franks, Torous, 1994] авторы отмечают, что издержки компаний, прошедших легальный процесс банкротства, больше на 4,5\%, чем у компаний, которые смогли справиться с финансовой несостоятельностью без вмешательства судебных инстанций. Опосредованно этот факт дает представление о мере прямых издержек финансовой неустойчивости, равных отмеченной разнице. В состав прямых издержек, по мнению Бранча, входят оплата услуг сторонних юристов, советников и т.д., специализирующихся на процессах банкротства. Обычно работа этих профессионалов очень высокооплачиваемая и, по мнению экспертов, занимает примерно $3-4,5 \%$ от первоначальной стоимости компании. Так, например, Вайс [Weiss, 1990] по выборке из 37 американских компаний получил цифру, равную $3,1 \%$ от суммарной стоимости балансового долга и рыночной стоимости акций. В обозначенной выше работе Альтмана прямые COFD составили в среднем 4,3\%, куда также входили прямые выплаты сторонним экспертам. Еще одно значение прямых COFD было представлено в исследовании Уорнера [Warner, 1977], где автор в ходе анализа 11 американских транспортных компаний оценил прямые COFD в размере 4,3\%. Что нам говорят эти цифры? Во-первых, можно отметить необыкновенную схожесть результатов исследований. На наш взгляд, это связано с возможностью непосредственно отследить прямые COFD через стоимость контрактов, заключаемых со сторонними профессионалами. Во-вторых, стоит отметить, что данные колеблются несущественно в течение более 25 лет и по разным отраслям, что указывает на их состоятельность. Очевиден, однако, и тот факт, что в предбанкротном состоянии менеджмент и персонал компании тратят намного большие усилия для вывода компании из затруднительного положения. Эти внутренние издержки, которые, в соответствии с исследованием Бранча, составляют 17-35\% от внешних издержек, должны быть включены в состав прямых издержек.

Анализ различных исследований относительно прямых COFD дает отчетливое представление об их размере. Тем не менее компании в финансово неустойчивом состоянии несут также и неявные издержки, которые, по мнению экспертов, довольно значительны и, судя по названию, трудны в исчислении. Действительно, если прямые COFD возникают непосредственно в процессе реорганизации, то неявные COFD могут влиять как до, так и после начала процесса. В первом случае они основаны на ожиданиях, во втором - на оценке 
упущенной выгоды. В рамках неявных COFD рассматривают потерянную ликвидность активов и, как следствие, потери от продажи активов по заниженным ценам на аукционах, потери рыночной доли и будущих операционных потоков, увеличение рискового спрэда требуемой ставки доходности и прочее. Более того, в исследовании Гилсона [Gilson, 1990] в состав неявных издержек включается возможность повторного прохождения процесса реорганизации (25\% рассмотренных фирм), однако конкретных цифр по COFD автор не предоставляет. В исследовании Бранча неявные COFD субъективно оцениваются в пределах $5-10 \%$, однако и здесь нам остается только довериться профессиональному чутью эксперта. Как бы то ни было, даже при отсутствии конкретных цифровых данных о размере неявных COFD существующие исследования позволяют сделать несколько полезных выводов, а именно:

1. Неявные COFD зависят от структуры долговых обязательств компании, и чем больше у компании кредиторов, тем выше транзакционные издержки урегулирования вопросов компенсационных выплат.

2. Согласно Гилсону, в США транзакционные издержки выше для фирм, которые решают проблему финансовой неустойчивости сами (reorganization out of court), чем для фирм, проходящих процедуру реорганизации в установленном законом порядке (filing for Chapter 11). Более того, фирмы, прошедшие через Chapter 11, имеют в среднем более низкое отношение долга к стоимости. Тем не менее подобная практика, скажем в России, может и не работать.

3. В работе Оплер и Титман [Opler, Titman, 1994] отмечают, что фирмы с высоким уровнем долга, а значит, высокими COFD, в состоянии финансовой неустойчивости теряют на 26\% больше рыночной доли, чем другие компании в аналогичной ситуации. Авторы особенно выделяют высокие риски для компаний, специализирующихся на специфичных активах.

Таким образом, даже на наиболее развитых рынках, где асимметрия информации меньше, а законодательство о банкротстве работает уже не первое десятилетие, издержки финансово несостоятельных компаний измеряются в двузначном процентном эквиваленте. Нам представляется, что в условиях развивающихся рынков, в том числе России, данные издержки принимают еще больший масштаб. Такое положение вещей ставит проблему определения влияния данного вида издержек на структуру капитала компании в ряд первостепенных задач. По сути, главной проблемой является даже не оценка размеров COFD, а оценка вероятности наступления кризисных явлений в компании. Маловероятно, что у фирмы есть какой-то определенный уровень долга, начиная с которого можно сказать, что компания подвержена расчетным значениям COFD, найденным выше. При этом более логично, что с повышением уровня долга растет не само абсолютное значение COFD, a именно вероятность банкротства. Эмпирические исследования Гилсона и Кала [Kahl, 2002], например, указывают на то, что даже после процесса реорганизации фактический уровень долга компании не меняется значительно, а значит, прямые издержки COFD незначительно зависят от уровня долга. В этом смысле эмпирическая проверка предположений компромиссной теории в разрезе издержек банкротства представляется более чем актуальной проблемой. Далее мы представим обзор наиболее значимых эмпирических моделей, связанных с издержками финансовой несостоятельности.

Наиболее важным моментом с точки зрения исследования является отбор объясняющих переменных (proxies), отражающих уровень издержек финансовой несостоятельности. Необходимо отметить, что здесь и в дальнейшем уровень COFD будет ассоциироваться исключительно с уровнем долгового финансирования; соответственно чем он выше, тем больше вероятность наступления финансовой несостоятельности и тем выше COFD. B работе Фамы и Френча [Fama\&French, 2002] основными переменными выступают уровень прибыльности, инвестиционные возможности фирмы и волатильность ожидаемых денежных потоков. Базовые предпосылки компромиссной модели исследователи интерпретировали следующим образом. По мнению авторов, ожидаемые издержки 
банкротства растут, когда прибыльность компании падает и это заставляет фирмы снижать долговой рычаг. В качестве показателя прибыльности рассматривается отношение EBIT/Total assets. Ожидаемые инвестиционные возможности фирмы рассматривается в работе как отношение рыночной стоимости к балансовой (market-to-book, V/A). В этом смысле авторы пытаются включить в анализ не только воспроизводимые активы (assets in place), но и возможности роста компании, а также нематериальные активы (intangibles), так как их стоимость заложена в рыночную стоимость компании. В качестве другого показателя инвестиционной активности компании авторы рассматривают темп роста ее активов. Рост активов - прямая оценка текущих инвестиций, и при условии постоянства уровня инвестиций он может рассматриваться как показатель ожидаемых темпов инвестирования. В этом смысле компания, которая расширяется (темп роста $>0$ ), и это расширение оценено рынком (V/A>1), менее подвержена потенциальному банкротству. Отдельного внимания заслуживает оценка волатильности денежных потоков компании. В своей работе авторы не прибегают к анализу исторических данных, так как непосредственная оценка волатильности ( $\sigma)$ даже на длительных временных промежутках сопряжена со значительным шумом оценки, поэтому в качестве объясняющей переменной авторы предлагают брать значение логарифма стоимости активов компании $(\ln \mathrm{A})$. Выбор поясняется тем, что большие компании обычно более диверсифицированы, а значит, общий уровень отклонений денежных потоков снижается с увеличением масштабов бизнеса. Соответственно ожидаемые издержки банкротства выше для компаний с большей волатильностью денежных потоков, что, в свою очередь, ведет к снижению уровня долга. В своей работе Фама и Френч на основе данных по 3000 фирм (COMPUSTAT) с 1965-го по 1999 год строят классическую множественную регрессионную модель, отражающую зависимость уровня долга от обозначенных выше параметров.

$$
\begin{aligned}
& L_{T+1} / A_{T+1}=b_{0}+b_{1} * V_{T} / A_{T}+b_{2} * E B I T_{T} / A_{T}+b_{3} * \ln \left(A_{T}\right)+e_{T}, \\
& \text { где } \\
& b_{0} \ldots b_{3}-\text { расчетные коэффициенты регрессии, } \\
& e_{T} \text { - случайная ошибка регрессии. }
\end{aligned}
$$

Как видно, уровень долгового финансирования в момент времени $\mathrm{T}+1$ зависит от переменных предыдущего периода Т. Другими словами, в модели наблюдается лаг во времени, равный одному периоду. Важно также отметить, что зависимой переменной выступает не рыночный уровень долга (L/V), а балансовое соотношение, а автор сознательно ставит знак тождества между этими значениями. Этот факт можно отнести к недостаткам модели, так как итоговое влияние на рыночные показатели может быть не таким однозначным. Тем не менее авторы делают вполне определенные выводы из модели:

1. Предпосылка о том, что более прибыльные фирмы прибегают к большему долговому финансированию, на практике не выполняется.

2. Предпосылка об отрицательной зависимости инвестиций компании и целевого уровня долга в целом подтверждается.

3. Модель фиксирует положительную зависимость между размером компании и уровнем долгового рычага. Таким образом, предположение о том, что наиболее крупные компании менее подвержены колебаниям денежных потоков, а значит, менее подвержены рискам финансовой несостоятельности, подтверждается.

Тем не менее авторы не преувеличивают значимости полученного результата, так как на соотношение D/V влияет множество факторов помимо волатильности денежных потоков. Например, крупным стабильным компаниям обычно упрощен доступ к финансовым ресурсам, а цена займа ниже, чем у малых фирм. Выводы данной модели подтверждаются и другими авторами, например, в исследовании Хомайфара [Homaifar et al.,1994]. Так, авторы на основе анализа более 400 американских фирм (данные Compustat за 1978-1988 годы) приходят к выводу, что размер фирмы $(\ln \mathrm{A})$ и возможности будущего роста компании (V/A) являются значимыми детерминантами структуры капитала. Расчетные значения, полученные 
для этих переменных, отражают положительную зависимость между размером фирмы и уровнем долга, а также сильную обратную связь между возможностями будущего роста и уровнем долга. В отличие от работы Фамы и Френча, авторы не отказываются от анализа исторических данных по колебаниям денежных потоков фирмы и получают сильную отрицательную связь волатильности и уровня долгового рычага. Рассмотренная выше работа Оплера и Титмана указывает на подверженность высокотехнологичных фирм рискам финансовой неустойчивости, где в качестве объясняющей переменной авторы выбирают отношение затрат на НИОКР к совокупным активам компании (RD/A). В исследовании Макки-Мейсона [MacKie-Mason, 1990] вероятность финансовой неустойчивости рассматривается через призму комплексного показателя финансовой стабильности ZАльтмана (ZPROB). В качестве одной из объясняющих переменных автор использует отношение 1/ZPROB, где фирмы с большим значением этого показателя более подвержены банкротству, а значит, и соответствующим издержкам, снижающим стоимость компании. Более того, автор углубляет анализ отклонений показателей чистой прибыли, оценивая не только абсолютные (VEARNA), но и процентные (VEARNB) колебания. На основе анализа 1500 фирм из базы данных Compustat за 1977-1987 годы было установлено, что все три показателя негативно связаны с уровнем долгового финансирования, что в целом подтверждает ранее сделанные предположения. В исследовании Олсона [Ohlson, 1980] автор в качестве оценки вероятности банкротства использует собственный О-счет, который представляет собой модифицированный Z-счет Альтмана с более детальным рассмотрением влияния денежных потоков и включением в анализ фиктивных переменных. Работа Саркара и Запатеро [Sarkar\&Zapatero, 2003] также фокусируется на анализе прибыльности компаний и влиянии прибыльности на структуру капитала. Авторы уделяют большое внимание изучению влияния эффекта разнонаправленного движения (mean reversion) денежных потоков компании и утверждают, что базовая компромиссная модель не выполняется, когда денежные потоки фирмы разнонаправлены во времени. Другими словами, эмпирический тест опять указывает на то, что более прибыльные компании не нацелены на высокий уровень заимствования, а следовательно, менее подвержены COFD. Также в работе подтверждается непосредственное влияние волатильности денежных потоков на рост долгового финансирования и вероятность наступления банкротства.

Что объединяет все рассмотренные эмпирические исследования? Прежде всего то, что они оценивают влияние тех или иных детерминантов на COFD исключительно в свете компромиссной теории структуры капитала. В этих работах авторы пытаются определить, соответствуют ли предпосылки модели trade-off действительности, то есть выполняется ли условие негативного влияния COFD на финансовый рычаг компании. В то же время мало внимания уделяется влиянию вероятности банкротства напрямую на стоимость компании, что, на наш взгляд, представляет наибольшую ценность. Этим проблемам посвящено исследование Чена и Мервиля [Chen, Merville, 1999], в котором авторы в качестве proxу для вероятности банкротства используют Z-счет Альтмана. Оценку потерь рыночной стоимости компаний авторы проводят через оценку потерь рыночной доли и возможностей роста инвестиций. Результатом анализа стало значение потерь в $8,3 \%$ от рыночной стоимости компании, попавшей в область финансовой несостоятельности. Таким образом, данное эмпирическое исследование в целом подтверждает представленные выше субъективные оценки различных авторов.

Итак, подводя итог вышесказанному, мы отмечаем серьезное влияние издержек финансовой несостоятельности на структуру капитала фирмы и потенциальные потери в стоимости компании. Консенсус-оценка суммарных COFD составляет $11-15 \%$ от общей стоимости компании до наступления финансовых затруднений. При этом авторы признают, что полученные значения могут быть и выше из-за фактической непредсказуемости поведения неявных COFD как до наступления банкротства, так и после него. Этот факт становится особенно значимым на фоне однородных результатов, полученных разными авторами для прямых COFD. В этом смысле мы видим дальнейшие перспективы 
исследований в области аппроксимации и более детальной оценки именно неявных издержек финансовой несостоятельности. Что касается построения статистических моделей, то ввиду неявности большей доли COFD влияние рассмотренных выше факторов на размер COFD и потери в стоимости фирмы оцениваются опосредованно через уровень долга. Другими словами, принимая эти условия, мы принимаем предпосылки компромиссной модели, выполнимость которой в реальной экономике многими исследователями также ставится под сомнение.

\section{Список литературы}

1. Altman E.I. The Success of Business Failure Prediction Models / Journal of Banking and Finance, No. 8, 1984, p. 171-198.

2. Branch B. The Cost of Bankruptcy. A Review / International Review of Financial Analysis, No. 11, 2002, p. 39-57.

3. Chen G.M.; Merville L.J. An Analysis of the Underreported Magnitude of the Total Indirect Costs of Financial Distress / Review of Quantitative Finance and Accounting, No. 13, 1999, p. $277-293$.

4. Fama E.F.; French K.R. Testing Trade-Off and Pecking Order Predictions about Dividends and Debt / The Review of Financial Studies, No. 15, 2002, p. 1-33. http://www.jstor.org/stable/2696797.

5. Franks J.; Torous W. A comparison of financial restructuring is distress exchanges and Chapter 11 reorganizations / Journal of Financial Economics, No. 12, 1994, p. 349-370.

6. Gilson S.C. Management turnover and financial distress / Journal of Financial Economics, No. 25, 1990, p. 241-262.

7. Homaifar G.; Zietz J.; Benkato O. An Empirical Model of Capital Structure: Some New Evidence / Journal of Business Finance \& Accounting, No. 21, 1994.

8. Kahl M. Economic Distress, Financial Distress, and Dynamic Liquidation / The Journal of Finance, No. 57, 2002, p. 135-168. http://www.jstor.org/stable/2697836.

9. MacKie-Mason J.K. Do Taxes Affect Corporate Financing Decisions? / The Journal of Finance, No. 45, 1990, p. 1471-1493. http://www.jstor.org/stable/2328746.

10. Ohlson J.A. Financial Ratios and the Probabilistic Prediction of Bankruptcy / Journal of Accounting Research, No. 18, 1980, p. 109-131. http://www.jstor.org/stable/2490395.

11. Opler T.C.; Titman S. Financial Distress and Corporate Performance / The Journal of Finance, No. 49, 1994, p. 1015-1040. http://www.jstor.org/stable/2329214.

12. Sarkar S.; Zapatero F. The Trade-Off Model With Mean Reverting Earnings: Theory And Empirical Tests / The Economic Journal, No. 113, 2003, p. 834-860.

13. Warner J.B. Bankruptcy Costs: Some Evidence / The Journal of Finance, No. 32, 1976, p. 337-347. http://www.jstor.org/stable/2326766.

14. Weiss L. Bankruptcy resolution: direct costs and violation of priority claims / Journal of Financial Economics, No. 27, 1990, p. 285-314. 